\title{
More (Adjustment) Is Not Always Better: How Directed Acyclic Graphs Can Help Researchers Decide Which Covariates to Include in Models for the Causal Relationship between an Exposure and an Outcome in Observational Research
}

\author{
Elizabeth W. Diemer ${ }^{a}$ James I. Hudson ${ }^{b, c}$ Kristin N. Javaras ${ }^{b, c}$ \\ a Department of Child and Adolescent Psychiatry, Erasmus Medical Center, Rotterdam, The Netherlands; \\ ${ }^{b}$ McLean Hospital, Belmont, MA, USA; ${ }^{C H a r v a r d}$ Medical School, Boston, MA, USA
}

\begin{abstract}
Keywords
Causal inference · Bias · Directed acyclic graph · DAG ·

Methods
\end{abstract}

\section{Introduction}

When constructing a model for an outcome of interest (e.g., a linear regression model), the choice of covariates to be included depends in part on the researcher's aims. (Although the term covariate has been variously defined, here we simply use covariate to refer to an explanatory variable included in a regression model that is not the explanatory variable of interest, i.e., the exposure.) If the aim is to build a predictive model [1], covariate selection focuses on improving model predictions while also limiting overfitting, which occurs when an overly complex model yields predictions that are too specific to a particular dataset, reducing its generalizability $[2,3]$. Alternatively, the aim may be to build a causal model [1]. (Note that Table 1 contains a glossary with definitions for all key terms, which are italicized.) For instance, one might build a causal model in order to quantify the average causal effect of an exposure on an outcome. The average causal

karger@karger.com www.karger.com/pps

Karger ${ }^{\prime \prime} \div$

BOPEN ACCESS
(C) 2021 The Author(s)

Published by S. Karger AG, Basel

This is an Open Access article licensed under the Creative Commons Attribution-NonCommercial-4.0 International License (CC BY-NC) (http://www.karger.com/Services/OpenAccessLicense), applicable to the online version of the article only. Usage and distribution for commercial purposes requires written permission. effect can be defined as the average difference in outcome had each individual in the population experienced the exposure, as compared to if no one had experienced the exposure [4]. As an illustration for this commentary, we will consider a causal model of the effect of the exposure preterm birth on the outcome attention-deficit/hyperactivity disorder (ADHD) during childhood. Because this example is offered purely for illustrative purposes, we have deliberately simplified it and ignored methodological difficulties that would complicate a real-world investigation of this issue (e.g., mismodeling the functional form of the variables, measurement error, and presence of unmeasured confounders).

With causal models, the aim is to obtain the least biased possible estimate of the average causal effect, where the term "bias" is used to mean any deviation from an accurate measurement [5]. Thus, covariate selection should focus on eliminating or reducing bias. One type of bias occurs when the estimated causal effect reflects not only the causal relationship between the exposure and the out-

Elizabeth W. Diemera: Work completed while a student at the Harvard School of Public Health, Boston, MA, USA. 
Table 1. Glossary of key terms

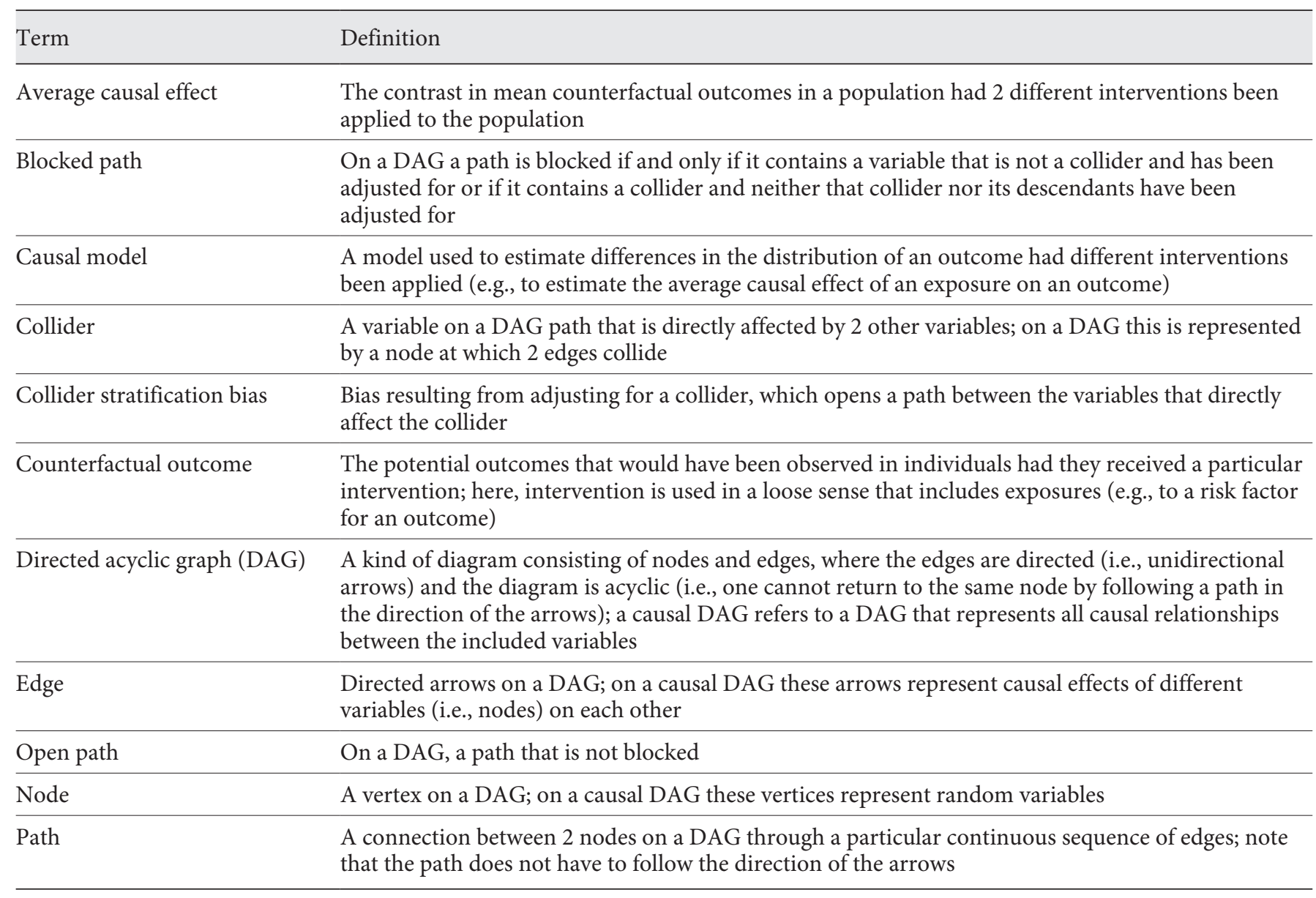

come but also other relationships between the exposure and the outcome [6], as is typically the case in observational studies [7]. In our example, the unadjusted association between preterm birth and ADHD may be a biased estimate of the causal effect of interest if there are other variables that link preterm birth and ADHD, aside from the intermediate variables that mediate the exposure's causal effect on the outcome (i.e., variables in the causal pathway between preterm birth and ADHD).

When selecting covariates, researchers may choose to include certain variables because they believe them to be potential confounders (i.e., common causes of the exposure and outcome) that, if not included in the model, will lead to biased estimates of the average causal effect. For example, researchers might adjust for maternal smoking during pregnancy because they believe that it has a causal effect on both preterm birth and ADHD. If this were the case, then unadjusted estimates of the association between preterm birth and ADHD would be biased, because these estimates would reflect not only the association due to the causal effect of preterm birth on ADHD but also the association due to the common effects of maternal smoking during pregnancy on exposure and outcome. Additionally, researchers at times choose to include certain covariates because existing research has identified them as risk factors for the outcome, or as correlates of the exposure. However, adjusting for covariates can, in certain circumstances, do more harm than good by introducing new bias into an estimate of the causal effect $[8,9]$.

To examine the potential perils of covariate adjustment we provide an introduction to causal directed acyclic graphs (DAGs). Causal DAGs can clarify how adjustment for a given covariate might impact bias, by providing a simple way to visualize assumptions about the statistical relationships between the exposure, outcome, and covariates in question. We illustrate their use with the example of preterm birth and ADHD. 
Fig. 1. Example of DAG for the causal effect of child preterm birth on child ADHD. Variables are represented by nodes on a DAG. Assumed causal relationships between variables are represented by directed edges (i.e., arrows) between nodes. Typically, variables later in terms of temporal order appear farther to the right.

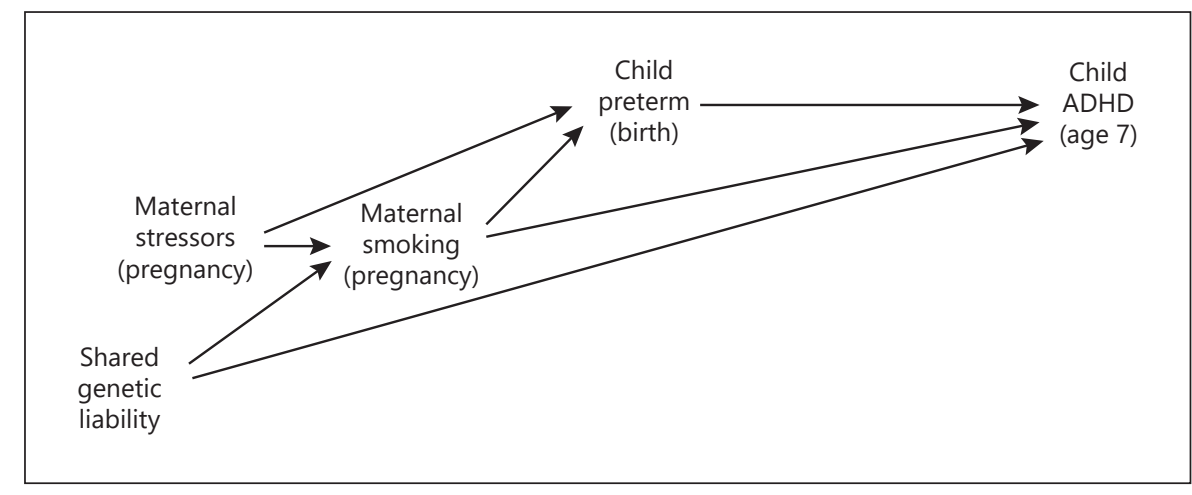

\section{An Introduction to DAGs}

The development and use of DAGs to clarify causal models in epidemiologic research has been described elsewhere [10-12]. Causal DAGs are a kind of causal diagram consisting of nodes, which represent variables, and directed edges (i.e., arrows), which represent causal relationships between variables. As an example, see Figure 1, which presents a DAG for a simplified causal model for preterm birth and ADHD, which we consider in detail in the next section. An arrow on a DAG does not imply that there is definitely a causal relationship between the 2 variables but only that there might be a relationship between them. In contrast, the absence of an arrow between 2 variables implies that the researcher is certain that one variable does not cause the other. These graphs are acyclic, which means that, if one follows the direction of arrows on a DAG, no path from a variable will lead back to itself.

Researchers can apply several graphical rules to DAGs to help decide which, if any, covariates to include in the causal model (Fig. 2). These rules relate to paths between the variables depicted in the graph. A path is a connection between 2 variables on a DAG through a particular continuous sequence of arrows. Note that the presence of a path through a series of variables is independent of the directions of the arrows involved in the path and does not imply any particular type of association between those variables. In other words, a path does not need to follow the direction of the arrows involved (e.g., in Fig. 1, there is a path between preterm birth and ADHD that passes through maternal smoking during pregnancy). However, the direction of the arrows does affect whether a path is open or blocked. An open path can be thought of as a path along which one can travel unimpeded, in the sense that one does not encounter blocks at any of the nodes between the endpoints; determining whether a path is blocked at a particular node involves the application of several rules that are described in the following paragraph. Notably, open paths imply statistical associations between the endpoint variables, whereas blocked paths imply the absence of an association. If a path is open, then it will contribute to the estimated association between the variables on the 2 ends of the path. Thus, aside from paths representing the causal effect of interest (e.g., paths from preterm birth to ADHD), all other paths between the exposure and outcome will ideally be blocked. If not, then the resulting estimate of the average causal effect will be biased, although the magnitude of this bias will vary depending on the strength and nature of the causal relationships represented in the other open paths, as we illustrate in the following section. Of course, it is possible that all other open paths between exposure and outcome might cancel each other exactly, meaning that the net bias due to other open paths would be null. However, such balancing is empirically untestable and seems unlikely to occur in practice.

Whether a path is open or blocked depends on the relationships between the variables on that path and on which of those variables are included as covariates in the causal model. In a simple unadjusted model, paths between the exposure and outcome are open unless they pass through a collider (rule 1). A collider is a variable that is caused by the 2 adjacent variables along that path, as represented by edges pointing toward the variable. To understand why the presence of a collider blocks the path, suppose that maternal stressors during pregnancy and shared genetic liability both cause maternal smoking during pregnancy, which in turn causes both preterm birth and ADHD (Fig. 1). (Note that we use the variable name "shared genetic liability" as short-hand for overlapping genetic risk factors for maternal smoking during pregnancy and child ADHD.) Maternal smoking is a collider 
Fig. 2. Graphical rules for DAGs. For illustrative purposes, the blue arrow represents the relationship of interest (the causal effect of child preterm birth on child ADHD), and the letter $\mathrm{b}$ denotes the average causal effect one seeks to estimate. Also, in the examples, red is used to highlight how far one can travel along a given path from child preterm birth toward child ADHD before the path is blocked.
Rule 1: If no variables are being conditioned on (i.e., adjusted for), the path between two variables is blocked if and only if the path moves through a pair of arrows pointing to the same variable. Such a variable is called a collider (because the two arrows collide on that variable). In the example illustrated below, maternal smoking is a collider on the path that goes through the nodes child preterm birth - maternal stressors - maternal smoking shared genetic liability - child ADHD. As a result, this path is blocked at maternal smoking, because it is a collider on that particular path. (Note that the same variable can be a collider on one path but not on a different path. For instance, maternal smoking is not a collider on the path that goes through the nodes child preterm birth maternal stressors - maternal smoking - child ADHD.)

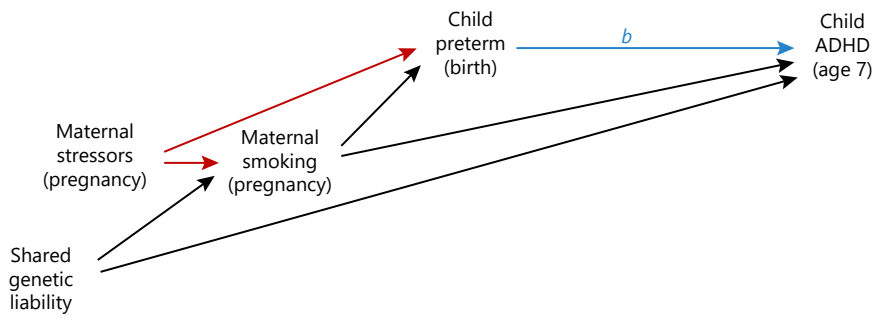

Rule 2: On a DAG, conditioning on (i.e., adjusting for) a variable is represented by drawing a box around a variable. Adjusting for a variable will block a path if that variable is not a collider on the path. In the example illustrated below, adjusting for maternal smoking blocks the path that goes through the nodes child preterm birth - maternal stressors - maternal smoking - child ADHD, because maternal smoking is not a collider on that path. Note that adjusting for a variable that is not a collider on a particular path will block that path, even if the variable is a collider on another path. For instance, adjusting for maternal smoking blocks the aforementioned path because maternal smoking is not a collider on that particular path, even though it is a collider on another path.
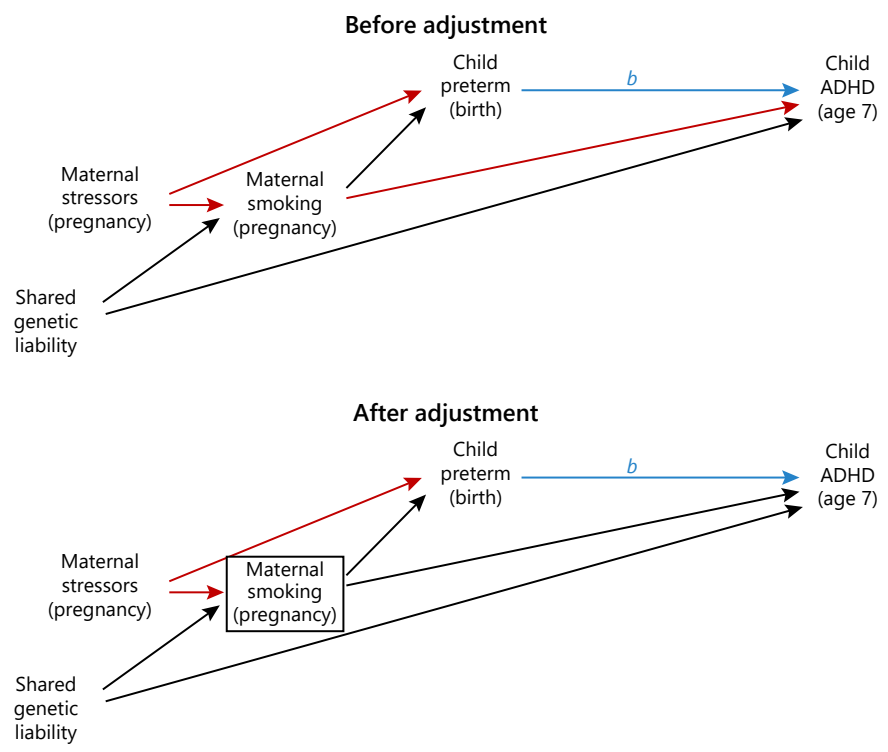

(Figure continued on next page.) on the path between preterm birth and ADHD that passes through both maternal stressors, maternal smoking, and shared genetic liability; this type of path is referred to as an M-shaped path (because it appears in the shape of the letter $\mathrm{M}$ when viewed sideways on our graph). Although maternal stressors can affect maternal smoking during pregnancy, this effect will not be transmitted to shared genetic liability because the latter is a cause, not an effect, of maternal smoking. Thus, the maternal smoking variable blocks this particular path.

Notably, adjusting for a variable can block a previously open path or open a previously blocked path, depending on the relationships between the variables in question. More specifically, adjusting for a variable that is not a collider on a particular path will block that path (rule 2). For example, if maternal smoking during pregnancy caused 


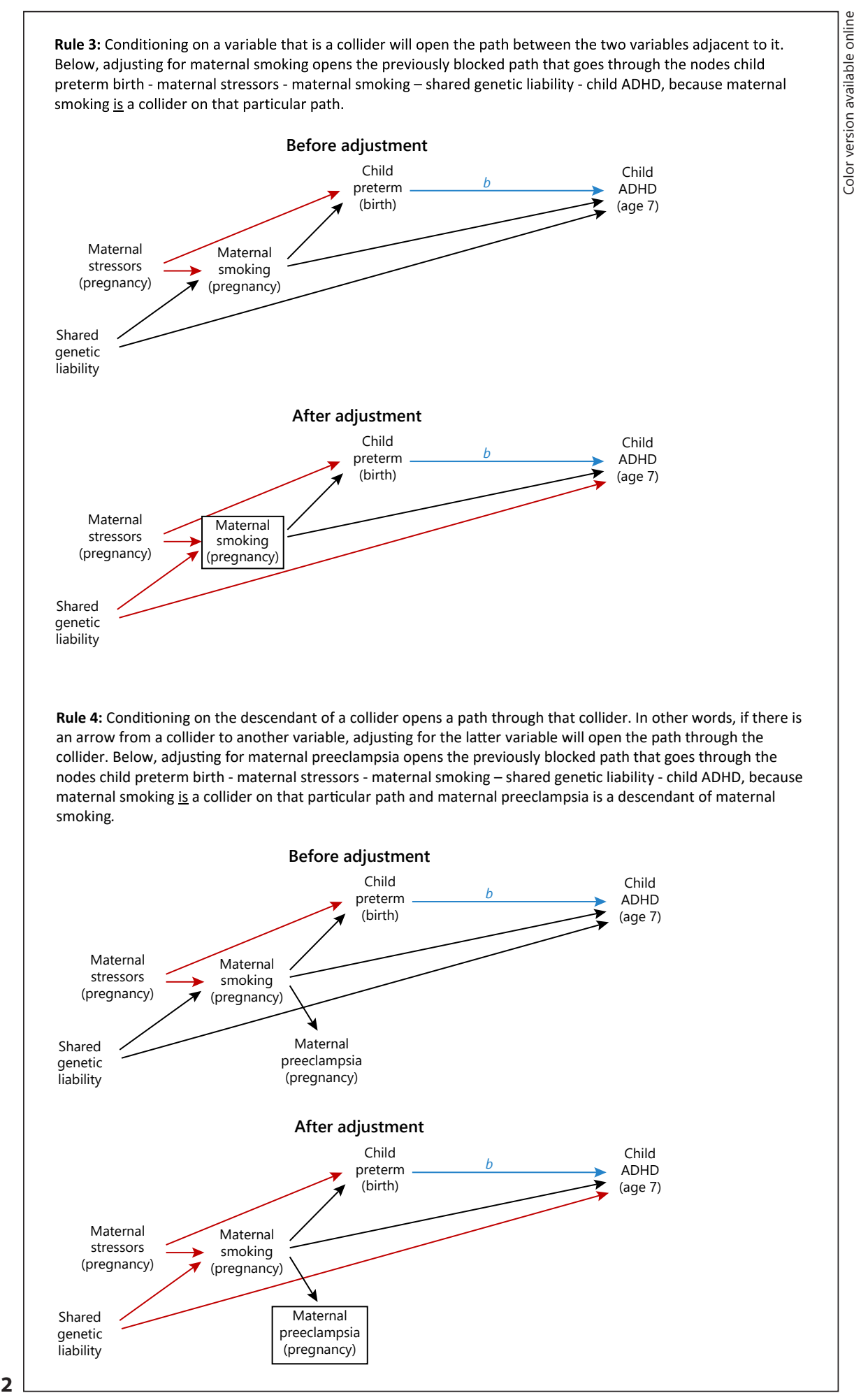


both child preterm birth and child ADHD, adjusting for maternal smoking would block the path from preterm birth to ADHD that goes through maternal smoking and represents confounding. In this instance, adjusting for maternal smoking would reduce bias due to confounding. However, adjusting for a variable that is a collider (rule 3) or its descendant (rule 4) opens the path previously blocked by that collider. To illustrate this, let us return to the example of maternal smoking. Since it is a collider on the aforementioned $\mathrm{M}$-shaped path between preterm birth and ADHD, that path is blocked without adjusting for maternal smoking in the causal model (rule 1). In fact, adjusting for maternal smoking would actually induce a relationship between shared genetic liability and maternal stressors, thereby opening the M-shaped path between preterm birth and ADHD and introducing bias into the estimate of the average causal effect. This bias can be positive or negative and is referred to as collider stratification bias (the more general term) or M-bias (a specific type of collider stratification bias that occurs, e.g., when adjusting for a covariate that shares a common cause with the exposure and another common cause with the outcome) [8]. To understand how adjusting for a collider introduces bias, recall that adjusting for a variable amounts to holding it constant. At a constant level of maternal smoking, knowing the mother's stressors tells you something about their shared genetic liability, because maternal smoking is a function of shared genetic liability and maternal stressors. In this example, adjusting for maternal smoking both reduces bias (by blocking the path through maternal smoking that represents confounding) and introduces bias (by opening the M-shaped path through maternal smoking).

To eliminate bias due to other open paths between the exposure and outcome, the researcher must select a set of covariates such that all paths between the exposure and outcome are blocked, except for the path representing the causal effect of interest. Of course, in some situations (e.g., if the exposure is randomly assigned), no adjustment is needed because there are no longer any paths that have edges directed to the exposure because the only cause of the exposure is the random process. Alternative$\mathrm{ly}$, in other situations, even if every variable were measured, there is no set of covariates that can block all other paths. These types of situations necessitate using more complex methods of estimation [13, 14], which require their own similarly strong assumptions. These complex methods can also be useful in situations where there is a set of covariates that block all other paths, but some or all of the covariates in that set have not been measured [15].

\section{Illustrative Example}

We now return to our example of preterm birth and ADHD. By way of background, the potential effect of preterm birth on ADHD has received considerable attention from researchers [16-20]. Most studies have documented a positive association between the 2 variables, and various causal mechanisms have been proposed [21, 22]. However, the interpretation of these findings is complicated by the researchers' choice of covariates, since adjusting for additional variables in the causal model could introduce bias, depending on those variables' actual relationship to preterm birth and ADHD. As noted above, researchers often choose to include certain covariates because they believe that they may confound the exposure-outcome relationship. For example, maternal smoking during pregnancy has been considered a potential confounder [16], leading to its inclusion as a covariate in models used to examine the relationship between preterm birth and ADHD [17, 18, 20]. However, although there is a consistent association between maternal smoking during pregnancy and child ADHD [23], maternal smoking is not necessarily a cause of ADHD; instead, the association may reflect a common cause, such as a shared genetic liability [24].

Since our example is intended for didactic purposes, we introduce several simplifying assumptions, beginning with the assumption that relevant constructs (e.g., ADHD in childhood and maternal smoking) can be measured by single variables. Critically, we assume that the DAG depicted in Figure 1 includes all paths between preterm birth and ADHD, including paths that pass through maternal smoking during pregnancy (a potential confounder). We also assume that maternal stressors during pregnancy and shared genetic liability have not been measured in the study, and it is thus not possible to include them as covariates. We recognize that, in practice, these assumptions are unlikely to be met, and thus our example is not meant to be taken as a valid analysis of the actual causal effects involved.

The DAG in Figure 1, which represents the assumed causal relationships among the variables involved, depicts several pathways by which maternal smoking could relate to preterm birth and ADHD. One of these ways is as a confounder, i.e., a cause of both preterm birth and ADHD. However, maternal smoking could also relate to the exposure and outcome through common causes (e.g., maternal stressors during pregnancy and shared genetic liability, respectively). Depending on which of these paths is present, including maternal smoking as a covariate (i.e., 
Fig. 3. Two scenarios for how maternal smoking during pregnancy relates to child preterm birth and to child ADHD. It is not standard practice to include numeric values corresponding to paths; however, we do so in $\mathbf{a}$ and $\mathbf{b}$ for illustrative purposes, to highlight the differing strength of the relationships depicted in the 2 contrasting scenarios. The magnitude of the value appearing next to an edge reflect the strength of the (directed) relationship between the 2 variables connected by the edge in the model used to generate simulated data consistent with the DAG.
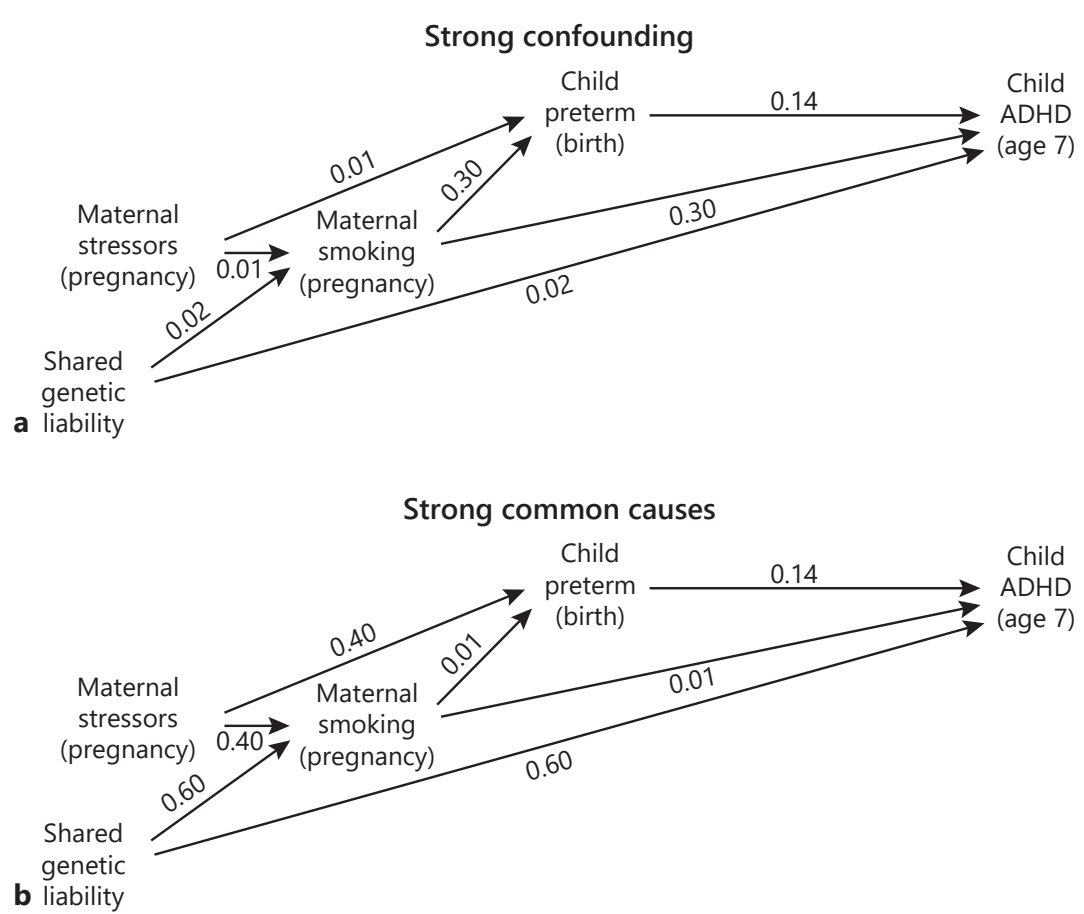

in the causal model for the effect of preterm birth on ADHD) could increase bias, reduce bias, or both.

Applying the graphical rules presented in Figure 2 to the question of how to handle maternal smoking, we can see that, without adjusting for maternal smoking, there are 3 open paths between preterm birth and ADHD aside from the path representing the causal effect of preterm birth on ADHD. These other open paths between preterm birth and ADHD, which represent confounding, include the path that goes through maternal smoking only, the one that goes through both maternal stressors and maternal smoking, and the one that goes through both maternal smoking and shared genetic liability. Due to these open paths through maternal smoking, we can expect that unadjusted estimates of the average causal effect (of preterm birth on ADHD) will be biased. Including maternal smoking as a covariate in our causal model will block these paths, eliminating these particular sources of bias. However, adjusting for maternal smoking will also introduce collider stratification bias, as noted above. This happens because maternal smoking is a collider on the M-shaped path that connects preterm birth and ADHD via maternal stressors, maternal smoking, and shared genetic liability.

Whether blocking the 3 previously open paths or opening the 1 previously blocked path introduces more bias depends on the strengths of the relationship between the variables on these paths through maternal smoking. To illustrate this point, we performed simulations for 2 scenarios where the strengths of these relationships differ markedly (note that the selected values of the parameters were not based on actual empirical evidence from the literature but rather were chosen to illustrate scenarios for didactic purposes). In these simulations, we make several additional simplifying assumptions, including assumptions that the models in our simulations are correctly specified and that predictors (e.g., preterm birth) can be measured without error. In reality, violations of these assumptions may represent additional sources of bias.

All simulations were performed using R for MAC OS $\mathrm{X}$ version 3.4.4 [25]. (See online suppl. Appendix A for code; see www.karger.com/doi/10.1159/000517104 for all online suppl. material) For the first and second scenarios, we generated 10,000 datasets (with $n=250$ ) consistent with the DAGs depicted in Figure $3 \mathrm{a}$ and b, respectively. More specifically, datasets were generated under the assumption of normality for each exogenous variable and the standard assumptions of linear regression (linearity, independent observations, homoskedasticity, and normality of errors) for the conditional distribution of each endogenous variable. Further, in the models used to gen- 
erate the data, the means of exogenous variables were set equal to 0 , the coefficients of the linear regression models (used to generate the endogenous variables) were set equal to the numeric values appearing next to the relevant edges in Figure 3a (first scenario) or Figure 3b (second scenario), and the variances of all variables were set equal to 1 .

In the first scenario we examine (Fig. 3a), maternal smoking during pregnancy has strong effects on preterm birth and ADHD, reflecting a scenario where maternal smoking does confound the relationship between the two. Further, in this scenario, the unmeasured common causes (i.e., maternal stressors in pregnancy and shared genetic liability) have minimal effects on maternal smoking and preterm birth and on maternal smoking and ADHD, respectively. In this scenario, the unadjusted estimate (i.e., 0.23) of the coefficient for preterm birth (i.e., in a linear regression model for $\mathrm{ADHD}$ fitted to the simulated data) is heavily biased away from the true value $(=0.14)$, whereas the estimate adjusted for maternal smoking (i.e., 0.14) is effectively unbiased. This contrasts with the second scenario (Fig. 3b), where maternal smoking is a very weak confounder of the relationship between preterm birth and $\mathrm{ADHD}$, but the unmeasured common causes (i.e., maternal stressors during pregnancy and shared genetic liability) have strong effects on maternal smoking and preterm birth and on maternal smoking and ADHD, respectively. In this scenario, the estimate adjusted for maternal smoking (i.e., 0.08) is more biased than the unadjusted estimate (i.e., 0.15$)$, which is close to the true value $(=0.14)$ of the average causal effect of preterm birth on ADHD in our simulation. Notably, neither estimate in this scenario is as biased as the unadjusted estimate in the first scenario. These results are consistent with prior theoretical work [8] and simulation studies $[26,27]$ of the situation where a potential covariate is both a true confounder of the exposure-outcome relationship and also a collider on another path between the exposure and outcome. These studies suggest that the bias introduced by adjusting for that covariate could be less than the bias present in an unadjusted analysis unless the confounding effects are small compared to the effects of the unmeasured common causes.

\section{Discussion}

As the above example illustrates, when constructing a causal model, adjusting for a given variable can reduce bias, introduce bias, or both, depending on the true causal structure between the variables in question. If the potential covariate is both a confounder of the exposure- outcome relationship and a collider on another path between them, research suggests that the confounding bias eliminated by adjusting for the covariate will likely outweigh the collider stratification bias introduced by doing so, unless the confounding effects are small compared to the effects of the unmeasured common causes. Many real-world examples, including the relationship between preterm birth and ADHD, may more closely resemble the first scenario, meaning that adjusting for the potential confounder (e.g., maternal smoking) would result in less bias than failing to adjust for it. However, there are other situations where adjusting for a covariate is more likely to result in a net increase in bias. The most obvious situation is adjusting for a covariate that is an intermediate variable (i.e., a mediator) on one of the paths through which the exposure causes the outcome $[28,29]$. If that path contributes substantially to the average causal effect of interest, then adjusting for an intermediate covariate can introduce considerable bias. For example, researchers examining the relationship between preterm birth and ADHD might choose to adjust for perinatal hypoxicischemic events because they have been considered a potential risk factor for negative cognitive outcomes [30]. However, adjusting for hypoxic-ischemic events would bias estimates of the average causal effect of preterm birth on ADHD if hypoxic-ischemic events mediate at least some of this effect. More subtly, adjusting for a covariate that is caused by an intermediate variable, but not itself on the causal path between the exposure and outcome, can introduce bias when that covariate shares an unmeasured common cause with the outcome [28]. In this scenario, the degree of bias increases the stronger the relationship between the intermediate variable and the downstream covariate and the stronger the causal path through the intermediate variable [28]. In the above example, adjusting for low Apgar scores $[16,17,20]$ could bias estimates of the average causal effect of preterm birth on ADHD if low Apgar scores are caused by an intermediate variable (e.g., perinatal hypoxic-ischemic events) and also share other unmeasured common causes with child ADHD. Additionally, adjusting for a covariate that is an effect of the outcome can cause a net increase in bias, e.g., if the exposure has a direct effect (i.e., an effect not mediated via the outcome) on the covariate.

The challenges involved in deciding which covariates (if any) to include extends to many other situations where adjusting for particular covariates can either reduce or introduce bias, depending on the true causal structure [for examples, see 10,31-33]. Thus, the potential inclusion of any covariate in a causal model requires careful consider- 
ation. DAGs can facilitate this consideration by illuminating the assumed underlying causal structure of the variables under study and thereby revealing the consequences of adjusting for particular covariates under a variety of assumptions. Further, the use of DAGs becomes even more helpful when relationships are more complicated, since attempts to apply simple rules without graphical justification often fail completely in such instances.

Of note, selecting covariates and interpreting causal models requires not only careful consideration but also expert knowledge. Observational data alone are not typically sufficient to determine whether any given DAG is the true $\mathrm{DAG}$ or whether any given causal model is correct [9]. For instance, in our example, the potential covariate maternal smoking during pregnancy may be associated with the outcome ADHD because: the former causes the latter; both are caused by another, unmeasured variable (i.e., shared genetic liability); or both. We cannot determine from the dataset alone which of these is true and must instead make assumptions regarding the relationship between these variables. (There are a few special cases (e.g., the area of instrumental inequalities and the emerging area of causal discovery) in which it has been proposed that a particular DAG or causal model can be tested or falsified using observational data alone [34-36].) In common practice, both DAGs and causal models rely on the assumptions made by the researcher about the relationship of all measured and unmeasured variables in an analysis - assumptions that are typically based on previous research or knowledge of processes (e.g., biological processes) under investigation [9].

Despite the utility of causal DAGs, there are limitations that should be noted. To begin with, as illustrated in the above example, DAGs alone are insufficient to determine the direction or magnitude of bias introduced by adjustment, or lack of adjustment, for a covariate. Additionally, DAGs cannot depict the influence of effect measure modification (e.g., gender as a moderator of the effect of preterm birth on ADHD) [37], although there have been attempts to extend DAGs to consider such features. Additional potential limitations to the use of causal DAGs have been raised, including the possibility of oversimplification, but are beyond the scope of this commentary [38-40].

\section{Conclusion}

In observational psychiatric research, interest often lies in the causal relationship between an exposure and outcome. To estimate this relationship, researchers fit a causal model (i.e., a model for the relationship between the expo- sure and the outcome) to data. Researchers often include a variety of covariates in the model, typically in hopes of obtaining more accurate estimates of the causal effect of interest. However, as we have discussed and illustrate in an example, depending on the covariates' true relationships to the exposure and outcome, adjustment for covariates can actually introduce new bias instead of, or in addition to, reducing bias. Therefore, researchers should carefully consider which covariates to include in a causal model rather than simply including all covariates potentially related to the exposure or the outcome. DAGs are a useful tool for clarifying how covariate adjustment might impact bias in an estimate. By allowing researchers to visualize the assumptions required for a particular set of covariates to block all other paths between the exposure and outcome aside from the path of interest, DAGs allow researchers to contemplate whether those assumptions are reasonable.

\section{Acknowledgment}

The authors would like to acknowledge Dr. Kathryn McHugh, Dr. Linda Valeri, Dr. Emily Belleau, and Dr. Harrison Pope, Jr., for their very helpful feedback on earlier drafts of this paper.

\section{Conflict of Interest Statement}

During the past 3 years, K.N. Javaras has received research grant support from the National Institute of Diabetes and Digestive and Kidney Disease, an Eleanor and Miles Shore Harvard Medical School Fellowship, and a McLean Hospital Jonathan Edward Brooking Mental Health Research Scholar Award. She also owns stock in Sanofi and the Centene Corporation. J.I. Hudson has received consulting fees from Idorsia, PPD Development, Shire, and Sunovion and has research grant support from Boehringer Ingelheim, Idorsia, and Sunovion. E.W. Diemer has no conflict of interests to report.

\section{Funding Sources}

The work of K.N. Javaras on this project was supported by K23DK120517 and the McLean Hospital Women's Mental Health Innovation Fund. E.W. Diemer is supported by an innovation program under Marie Sklodowska-Curie grant agreement No. 721567. No funding source had any role in the design, collection, analysis, writing, or decision making of this study.

\section{Author Contributions}

K.N. Javaras and E.W. Diemer conceived this work and drafted the original version. J.I. Hudson critically revised this work. All of the authors approved the final version of this paper. 


\section{References}

1 Shmueli G. To explain or to predict? Stat Sci. 2010;25(3):289-310.

2 Mullainathan S, Spiess J. Machine learning: an applied econometric approach. J Econ Perspect. 2017;31(2):87-106.

3 Lever J, Krzywinski M, Altman N. Points of significance: Model selection and overfitting. Nature Methods. 2016;13(9):703-4

4 Robins JM. Data, design, and background knowledge in etiologic inference. Epidemiology. 2001 May;12(3):313-20.

5 Rothman KJ, Greenland S, Lash TL. Modern epidemiology. Third edition. Philadelphia: Lippincott Williams \& Wilkins; 2008.

6 Rothman KJ. Epidemiology: An introduction. Second edition. Oxford: Oxford University Press; 2012.

7 Ohlsson H, Kendler KS. Applying causal inference methods in psychiatric epidemiology: a review. JAMA Psychiatry. 2020 Jun;77(6):637-44.

8 Greenland S. Quantifying biases in causal models: classical confounding vs colliderstratification bias. Epidemiology. 2003 May;14(3):300-6.

9 Greenland S, Robins JM, Pearl J. Confounding and collapsibility in causal inference. Stat Sci. 1999;14(1):29-46.

10 Hernán MA, Hernández-Díaz S, Werler MM, Mitchell AA. Causal knowledge as a prerequisite for confounding evaluation: an application to birth defects epidemiology. Am J Epidemiol. 2002 Jan;155(2):176-84.

11 Shrier I, Platt RW. Reducing bias through directed acyclic graphs. BMC Med Res Methodol. 2008 Oct; $8(1): 70$.

12 Glymour MM, Greenland S. Causal diagrams. In: Rothman KJ, Greenland S, Lash TL, editors. Modern epidemiology. Third edition. Philadelphia: Lippincott, Williams \& Wilkins 2008. pp. 183-209.

13 Robins JM, Hernán MA, Brumback B. Marginal structural models and causal inference in epidemiology. Epidemiology. 2000 Sep;11(5):550-60.

14 Robins JM. The analysis of randomized and non-randomized AIDS treatment trials using a new approach to causal inference in longitudinal studies. In: Sechrest L, Freeman H, Mulley A, editors. Health service research methodology: A focus on AIDS. Washington: National Center for Health Services Research; 1989. p. 113-59.
15 Hernán MA, Robins JM. Instruments for causal inference: an epidemiologist's dream? Epidemiology. 2006 Jul;17(4):360-72.

16 Halmøy A, Klungsøyr K, Skjærven R, Haavik J. Pre- and perinatal risk factors in adults with attention-deficit/hyperactivity disorder. Biol Psychiatry. 2012 Mar;71(5):474-81.

17 Lindström K, Lindblad F, Hjern A. Preterm birth and attention-deficit/hyperactivity disorder in schoolchildren. Pediatrics. 2011 May;127(5):858-65.

18 Sucksdorff M, Lehtonen L, Chudal R, Suominen A, Joelsson P, Gissler M, et al. Preterm birth and poor fetal growth as risk factors of atention-deficit/hyperactivity disorder. Pediatrics. 2015 Sep;136(3):e599-608.

19 Schieve LA, Tian LH, Rankin K, Kogan MD, Yeargin-Allsopp M, Visser S, et al. Population impact of preterm birth and low birth weight on developmental disabilities in US children. Ann Epidemiol. 2016 Apr;26(4):267-74.

20 Silva D, Colvin L, Hagemann E, Bower C. Environmental risk factors by gender associated with attention-deficit/hyperactivity disorder. Pediatrics. 2014 Jan;133(1):e14-22.

21 Murray E, Pearson R, Fernandes M, Santos IS Barros FC, Victora CG, et al. Are fetal growth impairment and preterm birth causally related to child attention problems and ADHD? Evidence from a comparison between highincome and middle-income cohorts. J Epidemiol Community Health. 2016 Jul;70(7):7049

22 Sciberras E, Mulraney M, Silva D, Coghill D. Prenatal risk factors and the etiology of $\mathrm{AD}$ HD-Review of existing evidence. Curr Psychiatry Rep. 2017 Jan;19(1):1.

23 Huang L, Wang Y, Zhang L, Zheng Z, Zhu T, $\mathrm{Qu} \mathrm{Y}$, et al. Maternal smoking and attentiondeficit/hyperactivity disorder in offspring: a meta-analysis. Pediatrics. 2018 Jan;141(1) e20172465.

24 Knopik VS. Maternal smoking during pregnancy and child outcomes: real or spurious effect? Dev Neuropsychol. 2009;34(1):1-36.

25 R Core Team. R: A Language and Environment for Statistical Computing. Vienna, Austria: R Foundation for Statistical Computing; 2020.

26 Ding P, Miratrix LW. To adjust or not to adjust? Sensitivity analysis of M-bias and butterfly-bias. J Causal Inference. 2015;3(1):41-57.
27 Liu W, Brookhart MA, Schneeweiss S, Mi X, Setoguchi S. Implications of $\mathrm{M}$ bias in epidemiologic studies: a simulation study. Am I Epidemiol. 2012 Nov;176(10):938-48.

28 Schisterman EF, Cole SR, Platt RW. Overadjustment bias and unnecessary adjustment in epidemiologic studies. Epidemiology. 2009 Jul;20(4):488-95.

29 Kaufman JS. Statistics, adjusted statistics, and maladjusted statistics. Am J Law Med. 2017 May;43(2-3):193-208.

30 Galinsky R, Lear CA, Dean JM, Wassink G, Dhillon SK, Fraser M, et al. Complex interactions between hypoxia-ischemia and inflammation in preterm brain injury. Dev Med Child Neurol. 2018 Feb;60(2):126-33.

31 Hudson JI, Javaras KN, Laird NM, VanderWeele TJ, Pope HG Jr, Hernán MA. A structural approach to the familial coaggregation of disorders. Epidemiology. 2008 May;19(3): 431-9.

32 Banack HR, Kaufman JS. The "obesity paradox" explained. Epidemiology. 2013 May;24(3):461-2.

33 Hernández-Díaz S, Schisterman EF, Hernán MA. The birth weight "paradox" uncovered? Am J Epidemiol. 2006 Dec;164(11):1115-20.

34 Malinsky D, Danks D. Causal discovery algorithms: A practical guide. Philos Compass. 2018 Jan;13(1):e12470.

35 Richardson TS. A discovery algorithm for directed cyclic graphs. arXiv preprint. arXiv: 1302.3599, 2013.

36 Pearl J. On the testability of causal models with latent and instrumental variables. arXiv preprint. arXiv: 1302.4976, 2013.

37 VanderWeele TJ, Robins JM. Directed acyclic graphs, sufficient causes, and the properties of conditioning on a common effect. Am J Epidemiol. 2007 Nov;166(9):1096-104.

38 Dawid AP. Beware of the DAG! In: Causality: Objectives and assessment. Available from http://proceedings.mlr.press/v6/dawid10a. html.

39 Rubin D. Author's reply (to Ian Shrier's letter to the editor). Stat Med. 2008;27(14):2741-2.

40 Krieger N, Davey Smith G. The tale wagged by the DAG: broadening the scope of causal inference and explanation for epidemiology. Int J Epidemiol. 2016 Dec;45(6):1787-808. 